\title{
Avaliação do conhecimento de agentes comunitários de saúde acerca da hanseníase em um município hiperendêmico
}

\author{
Nayane Batista de Melo ${ }^{a}$, Stefane Vieira Nobre ${ }^{b, *}$, Bruna Lorena Oliveira Souza ${ }^{a}$, Lucas \\ Dias Soares Machado ${ }^{\mathrm{b}}$, Thiáskara Ramile Caldas Leite ${ }^{\mathrm{a}}$
}

${ }^{\text {a }}$ Universidade Regional do Cariri - URCA, Crato, CE, Brasil

${ }^{\mathrm{b}}$ Universidade Estadual do Ceará - UECE, Fortaleza, CE, Brasil

\section{Histórico do Artigo: Recebido em: 26/03/2021 \\ Aceito em: 01/07/2021}

\section{Palavras-chave:} Conhecimento; Mycobacterium leprae; hanseníase; Atenção Primária à Saúde; Agentes Comunitários de Saúde.

Keywords:

Knowledge;

Mycobacterium leprae; leprosy; Primary Health Care; community health workers.

\begin{abstract}
RESUMO
A hanseníase é uma doença tropical negligenciada passível de identificação precoce e tratamento. Dos profissionais envolvidos em seu cuidado, destaca-se o Agente Comunitário de Saúde, que estabelece um vínculo intercambiável entre a unidade de saúde e o usuário. O objetivo deste estudo foi avaliar o conhecimento sobre hanseníase de agentes comunitários de saúde atuantes em município hiperendêmico. Trata-se de estudo transversal descritivo e quantitativo realizado com agentes comunitários de saúde de uma cidade do centro-sul cearense, no segundo semestre de 2017. Participaram 72 profissionais, respondentes de um questionário estruturado sobre principais aspectos requeridos na abordagem à doença. Realizou-se análise descritiva, através de distribuição de frequências absolutas e relativas, e inferencial, a partir de teste t e ANOVA de uma via. Obteve-se um nível de conhecimento ótimo para aspectos gerais e atribuições dos agentes comunitários de saúde, bom para os blocos tratamento e incapacidades físicas, regular para transmissão e diagnóstico e ruim para busca. Ao analisar o panorama geral do conhecimento das participantes, foi obtido um nível bom, correspondendo a $74 \%$ de assertivas corretas. Reconhece-se que os profissionais que têm/tiveram pacientes com hanseníase apresentam nível de conhecimento maior do que aqueles que não os têm. Evidencia-se um níve de conhecimento satisfatório das agentes comunitárias de saúde sobre a hanseníase. Entretanto deve-se atentar às necessidades de aprendizagem identificadas mediante as perguntas respondidas incorretamente, buscando o fomento de educação permanente junto aos profissionais do estudo, para que sua prática seja cada vez mais exitosa e respaldada em evidências atuais e vigentes.
\end{abstract}

Evaluation of the knowledge of community health workers about leprosy in a hyperendemic municipality

\begin{abstract}
Leprosy is a neglected tropical disease susceptible to early identification and treatment. Of the professionals involved in their care, the Community Health Workers stands out, which establishes an interchangeable link between the health unit and the user. The objective of this study was to evaluate the knowledge about leprosy of community health workers in a hyperendemic municipality. This is a descriptive and quantitative cross-sectional study carried out with community health workers from a city in the center-south of Ceará, in the second semester of 2017. 72 professionals participated, responding to a structured questionnaire on the main aspects required to approach the disease. Descriptive analysis was carried out, through the distribution of absolute and relative frequencies, and inferential, using one-way $t$ test and ANOVA. An optimum level of knowledge was obtained for general aspects and attributions of community health workers, good for the treatment and physical disabilities, regular for transmission and diagnosis and poor for search. When analyzing the general panorama of the participants' knowledge, a good level was obtained, corresponding to $74 \%$ of correct statements. It is recognized that professionals who have / had leprosy patients have a higher level of knowledge than those who do not have them. There is evidence of a satisfactory level of knowledge of community health workers about leprosy. However, attention must be paid to the learning needs identified through the questions answered incorrectly, seeking to foster permanent education with the study professionals, so that their practice is increasingly successful and supported by current and current evidence.
\end{abstract}

\section{Introdução}

*Autor correspondente: stefanevn@outlook.com (Nobre S. V.) 
A hanseníase é uma doença infectocontagiosa, de evolução lenta causada pelo bacilo Mycobacterium Leprae, que tem alta infectividade, mas baixa morbidade. Manifesta-se, principalmente, por meio de sinais e sintomas dermatoneurológicos, mas pode afetar outros órgãos como o fígado, os testículos e os olhos $(1,2)$.

Apesar do avanço de estudos sobre formas de diagnóstico e terapêuticas, a hanseníase ainda é um problema de saúde pública mundial, principalmente em países em desenvolvimento como é o caso do Brasil (3). Neste sentido, a hanseníase constitui uma Doença Tropical Negligenciada (DTN), por ser intrinsecamente ligada a condições de pobreza e vulnerabilidade (4).

O Brasil é o único país da América Latina onde a doença não foi eliminada e o segundo lugar mundial de casos novos no ano de 2019, com 27.863 casos de hanseníase registrados (5). Sua distribuição é heterogênea entre as regiões brasileiras, estando predominantes as regiões Centro-Oeste, Norte e Nordeste (6).

A detecção precoce e realização do tratamento, a fim de evitar a transmissão da hanseníase entre indivíduos, são medidas importantes para o controle dessa doença. Nesse aspecto, destaca-se a Estratégia Saúde da Família (ESF) como porta de entrada do usuário no Sistema Único de Saúde (SUS) e que tem como premissa básica ações de prevenção e promoção da saúde, com importante participação do agente comunitário de saúde (ACS) $(7,8)$.

Essa categoria profissional mantém vínculos mais estreitos com a comunidade, estando apto a identificar precocemente as alterações no estado de saúde-doença-cuidado dos usuários (8). Para isto é necessário que o ACS detenha conhecimento sobre a doença e o seu curso, desempenhando seu papel adequado ao ofertar orientações corretas e identificar possíveis sinais e sintomas.

Assim, considerando o papel imprescindível do ACS no controle e na prevenção da hanseníase, se faz oportuno avaliar o conhecimento sobre a hanseníase entre os agentes comunitários de saúde atuantes em um município hiperendêmico.

\section{Materiais e Métodos}

Trata-se de um estudo transversal descritivo e analítico de abordagem quantitativa. $\mathrm{O}$ estudo teve como cenário macro o estado do Ceará, situado no nordeste brasileiro, e lócus de investigação o município de Iguatu, pertencente à região Centro-Sul do estado do Ceará. Configura-se como o principal polo econômico da região e também como cidade universitária (9) e, no ano de 2016, foi considerado um dos municípios prioritários para eliminação da hanseníase pela alta taxa de incidência da doença (10).

Participaram do estudo 72 profissionais ACS, vinculados a 19 equipes de saúde da família da zona urbana do município. Segundo dados do Departamento de Atenção Básica (11), em agosto de 2017, 29 Equipes de Saúde da Família estavam cadastradas no sistema, com uma proporção de cobertura populacional estimada em 98,08\%, atendendo a população das zonas urbana e rural.

Os participantes do estudo foram selecionados por meio de amostragem por conveniência sendo incluídos todos os ACS vinculados às equipes de saúde da família localizadas na zona urbana do município em estudo, mediante a leitura e assinatura do Termo de Consentimento Livre e Esclarecido (TCLE).

Os critérios de inclusão aplicados foram: ser ACS com, no mínimo, três meses de atuação na ESF, para garantir uma maior fidedignidade dos dados coletados. Este critério foi elencado, pois segundo dados da Coordenação da Atenção Básica, nos meses anteriores a coleta de dados, o município passou por um processo seletivo para 
contratação de ACS para áreas descobertas. Foram excluídos todos aqueles que se encontravam em regime de férias, licença ou atestado médico durante o período da coleta de dados.

Os dados foram coletados de setembro a outubro de 2017 e utilizou-se um questionário semiestruturado, elaborado pelos autores com base em manuais do Ministério da Saúde $(1,2)$. Era composto por questões sociodemográficas e profissionais dos participantes, tais como sexo, faixa etária, escolaridade, tempo de serviço, capacitações e existência de pacientes com a doença nas microáreas. Contemplava ainda variáveis, organizadas em blocos constituídos de cinco perguntas, acerca do conhecimento sobre aspectos gerais da doença, transmissão, diagnóstico, busca, tratamento, incapacidades físicas e atribuições do agente comunitário de saúde ao paciente com hanseníase.

Foi realizado um pré-teste com nove ACS para verificar a adequabilidade do instrumento, eliminando possíveis falhas da coleta de dados antes do início da pesquisa. O pré-teste serviu para testar o questionário em uma pequena amostra dos entrevistados, porém não houve a necessidade de modificações e adequações do questionário. Posteriormente ao pré-teste, os ACS que o responderam, foram excluídos da amostra.

O questionário foi entregue por dois pesquisadores aos participantes na unidade básica de saúde a qual estavam vinculados. Orientou-se que os ACS deveriam realizar o preenchimento na unidade, supervisionado por um pesquisador, em um tempo estimado de até 30 minutos. Os participantes foram orientados a não utilizar quaisquer meios de comunicação ou outras fontes de busca.

Os dados foram tabulados no Microsoft Office Excel® versão 2016 e analisados com auxílio do software Stata. Posteriormente foi realizada uma análise descritiva dos dados considerando suas frequências relativas e absolutas. Para análise das respostas referentes ao conhecimento, utilizou-se uma escala de acertos para mensuração do nível de conhecimento dos ACS sobre hanseníase, com base no número de respostas corretas obtidas, classificando-o em ótimo (90 a 100\%), muito bom (80 a 89\%), bom (70 a 79\%), regular (60 a 60\%), ruim (50 a 59\%) e muito ruim (abaixo de 50\%) (8).

De modo inferencial, foram realizadas análises de variância de uma via (ANOVA-One Way) com o intuito de avaliar se existiam diferenças no nível de conhecimento entre pessoas de estados civis diferentes (solteiras, casadas, em união estável e divorciadas), bem como entre pessoas de diferentes níveis de escolaridade. A normalidade dos dados foi averiguada segundo testes de Kolmogorov-Smirnov e Shapiro-Wilk. A homogeneidade da variância foi avaliada, enquanto pressuposto da ANOVA, por meio do teste de Levene e para a heterogeneidade identificada foi solicitada correção de Welch.

Realizou-se ainda, testes $t$ de Student para amostras independentes com o objetivo de analisar em que medida os níveis de conhecimento sobre hanseníase eram diferentes entre aqueles que participaram de capacitação sobre hanseníase, tem ou tiveram pacientes com hanseníase e os que cursaram a formação específica para ACS.

Foram realizados procedimentos de bootstrapping (1000 re-amostragens; 95\% IC BCa) para corrigir desvios da distribuição normal da amostra e tamanhos dos grupos, obter maior confiabilidade dos resultados e delimitar um intervalo de confianças de $95 \%$ para as diferenças entre as médias.

A pesquisa obedeceu aos preceitos éticos e legais dispostos pela resolução 466/2012 do Conselho Nacional de Saúde, apresentando adequação conforme aprovação pelo Comitê de Ética em Pesquisa da Universidade Regional do Cariri (CEP/URCA) sob o parecer número 2.358.416.

\section{Resultados}


A amostra foi composta por 72 ACS: mulheres com idade média de 40,64 anos $(\mathrm{DP}=10,27)$ e tempo de atuação médio de 11 anos $(\mathrm{DP}=8,94)$. Destas, 80,6\% ( $\mathrm{n}=58)$ participaram de formação específica para exercício da função de ACS, 69,4\% (n=50) receberam capacitação para enfrentamento da hanseníase e 76,4\% $(n=55)$ têm ou já tiveram pacientes com hanseníase em sua microárea de atuação. A tabela 1 complementa esta caracterização.

Tabela 1 - Caracterização sociodemográfica dos agentes comunitários de saúde e relacionada a capacitação sobre hanseníase e atendimento de paciente com hanseníase na microárea, Iguatu-Ceará, Brasil, 2017.

\begin{tabular}{|c|c|c|}
\hline Variavéis & $\mathbf{n}^{*}$ & $\%^{\dagger}$ \\
\hline \multicolumn{3}{|l|}{ Sexo } \\
\hline Feminino & 72 & 100 \\
\hline \multicolumn{3}{|l|}{ Faixa etária } \\
\hline $20-26$ & 03 & 4,2 \\
\hline $26-32$ & 11 & 15,3 \\
\hline $32-38$ & 19 & 26,3 \\
\hline $38-44$ & 13 & 18,1 \\
\hline $44-50$ & 10 & 13,9 \\
\hline $50-56$ & 09 & 12,5 \\
\hline $56-62$ & 05 & 6,9 \\
\hline $62-63$ & 02 & 2,8 \\
\hline \multicolumn{3}{|l|}{ Tempo de serviço } \\
\hline $0,25-4$ anos & 21 & 29,2 \\
\hline $4 \quad-8$ anos & 13 & 18,1 \\
\hline $8-12$ anos & 16 & 22,2 \\
\hline $12 \quad-16$ anos & 06 & 8,3 \\
\hline - 20anos & 03 & 4,2 \\
\hline $20-24$ anos & 05 & 6,9 \\
\hline $24 \quad-28$ anos & 05 & 6,9 \\
\hline $28-30$ anos & 03 & 4,2 \\
\hline \multicolumn{3}{|l|}{ Nível de escolaridade } \\
\hline Ensino fundamental completo & 08 & 11,1 \\
\hline Ensino médio incompleto & 02 & 2,8 \\
\hline Ensino médio completo & 47 & 65,3 \\
\hline Ensino superior incompleto & 06 & 8,3 \\
\hline Ensino superior completo & 09 & 12,5 \\
\hline \multicolumn{3}{|l|}{ Capacitações sobre hanseníase } \\
\hline Sim & 50 & 69 \\
\hline Não & 22 & 31 \\
\hline \multicolumn{3}{|c|}{ Pacientes com hanseníase na microárea } \\
\hline $\operatorname{Sim}$ & 55 & 76 \\
\hline Não & 17 & 24 \\
\hline Total & 72 & 100 \\
\hline
\end{tabular}

Nota: ${ }^{*}=$ frequência absoluta, ${ }^{\dagger}=$ frequência relativa

Os resultados referentes ao nível de conhecimento foram categorizados em sete blocos: bloco $\mathrm{A}$ - aspectos gerais da doença; bloco $\mathrm{B}$ - transmissão; bloco $\mathrm{C}$ - diagnóstico; bloco $\mathrm{D}$ - busca; bloco $\mathrm{E}$ - tratamento; bloco $\mathrm{F}$ - incapacidades físicas; e bloco $\mathrm{G}$ - atribuições 
dos agentes comunitários de saúde no cuidado ao paciente com hanseníase (Figura 1).

Figura 1 - Nível de conhecimento dos agentes comunitários de saúde sobre hanseníase. Iguatu, Ceará, 2017.

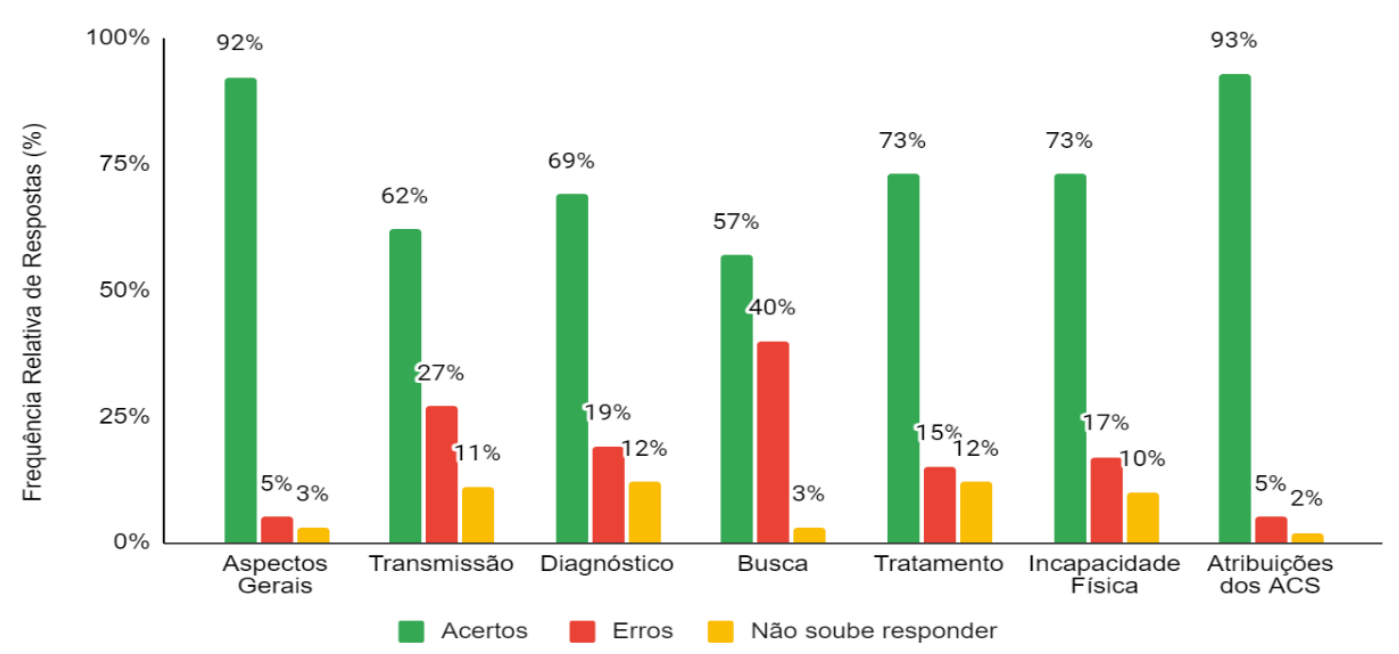

No bloco A, que trata sobre os aspectos gerais da doença, obteve-se que 94,4\% ( $\mathrm{n}=68$ ) das ACS reconheceram que a hanseníase atinge a pele e os nervos periféricos. Todas afirmaram que a doença tem cura e os principais sinais e sintomas são manchas esbranquiçadas, acastanhadas ou avermelhadas com diminuição da sensibilidade local.

Em relação à transmissão, avaliada no bloco $\mathrm{B}, 76,3 \%(\mathrm{n}=75)$ das ACS reconhecem que a transmissão ocorre através das vias aéreas superiores. 83,3\% $(\mathrm{n}=60)$ afirmam que o contato no ambiente familiar facilita o contágio da doença e $75 \%(n=54)$ acreditam que somente após os primeiros quinze dias da poliquimioterapia (PQT) o paciente não é mais fonte de transmissão.

Foram observadas $50 \%(\mathrm{n}=36)$ respostas incorretas e dúvidas quanto à única fonte de transmissão ser o homem e 76,38\% $(n=55)$ das ACS não sabiam se a transmissão da doença é restrita ao paciente multibacilar.

Quanto ao diagnóstico, abordado no bloco $\mathrm{C}, 72,2 \% \quad(\mathrm{n}=52)$ das participantes reconheceram que este é realizado por meio da história de vida do paciente e do exame dermatoneurológico, 81,9\% $(\mathrm{n}=59)$ indicaram que a realização do diagnóstico pode acontecer na unidade básica de saúde e $86,1 \%$ (n=62) afirmaram que a avaliação deve ser minuciosa pelas semelhanças com outras dermatoses. A baciloscopia de pele como exame complementar foi apontada por $82 \%(\mathrm{n}=59)$ dos participantes, entretanto, apenas $25 \%$ $(\mathrm{n}=18)$ sabem que a baciloscopia positiva classifica o paciente como multibacilar.

No bloco $\mathrm{D}$, que avaliou sobre busca, $58,3 \%(\mathrm{n}=42)$ das ACS afirmaram que cabe a elas a identificação e realização da notificação dos casos suspeitos de hanseníase. Reconhecem ainda, como sua responsabilidade, a execução de medidas de controle dos contatos e a monitorização vacinal de populações de risco, respectivamente com $43 \%$ $(n=31)$ e $68 \%(n=49)$ das respostas. Quanto à busca ativa dos casos, 61,1\% $(n=44)$ acreditam ser responsabilidade somente dos ACS, enquanto a busca passiva foi confirmada por $72,2 \%(n=52)$.

No bloco E, a respeito do tratamento, 97,2\% $(n=70)$ listaram que o tratamento é realizado na unidade básica de saúde de forma ambulatorial e gratuita, 98,6\% $(n=71)$ afirmaram que a adesão e realização de forma correta são fundamentais para eliminação 
da doença e 83,3\% ( $\mathrm{n}=60)$ reconhecem que o tratamento é realizado através da administração de doses supervisionadas na própria unidade e auto administradas no domicílio. No que diz respeito ao tempo de utilização das drogas para paucibacilares (PB) e multibacilares $(\mathrm{MB}), 63,8 \%(\mathrm{n}=46)$ responderam incorretamente ou não souberam e apenas 47,2\% $(n=34)$ conheciam os fármacos utilizados na PQT.

No bloco F, sobre incapacidades físicas, 97,2\% $(\mathrm{n}=70)$ das ACS responderam que há perda de sensibilidade nas pessoas com hanseníase, $88,8 \%(\mathrm{n}=64)$ reconheceram que o diagnóstico tardio é uma das causas de incapacidades físicas e 79,1\% $(n=57)$ relacionam o surgimento destas ao tratamento inadequado. Do total de ACS, 68\% ( $n=49)$ afirmaram que as incapacidades físicas decorrentes da hanseníase são mais comuns em pés, olhos e mãos e 36,1\% (n=26) relataram que as incapacidades físicas podem ser desenvolvidas mesmo após a cura.

Os itens do bloco $\mathrm{G}$, acerca das atribuições das ACS, demonstraram que reconhecem como suas funções: a realização de visitas domiciliares periódicas ao paciente com hanseníase $(100 \%, \mathrm{n}=72)$; a supervisão da tomada de medicação $(83,3 \%, \mathrm{n}=60)$; a identificação de sinais e sintomas e encaminhamento dos casos suspeitos para a unidade de saúde $(97,2 \%, \mathrm{n}=70)$; desenvolvimento de ações educativas na comunidade $(93 \%$, $\mathrm{n}=67$ ); e realização de orientações sobre a transmissão, autocuidado e combate aos estigmas vivenciados pelos pacientes $(93 \%, \mathrm{n}=67)$.

Os resultados apontam um nível de conhecimento ótimo para os blocos A (aspectos gerais) e $\mathrm{G}$ (atribuições dos agentes comunitários de saúde), bom para os blocos $\mathrm{E}$ (tratamento) e F (incapacidades físicas), regular para os blocos B (transmissão) e C (diagnóstico) e ruim para o bloco D (busca), como apresentado na Figura 2.

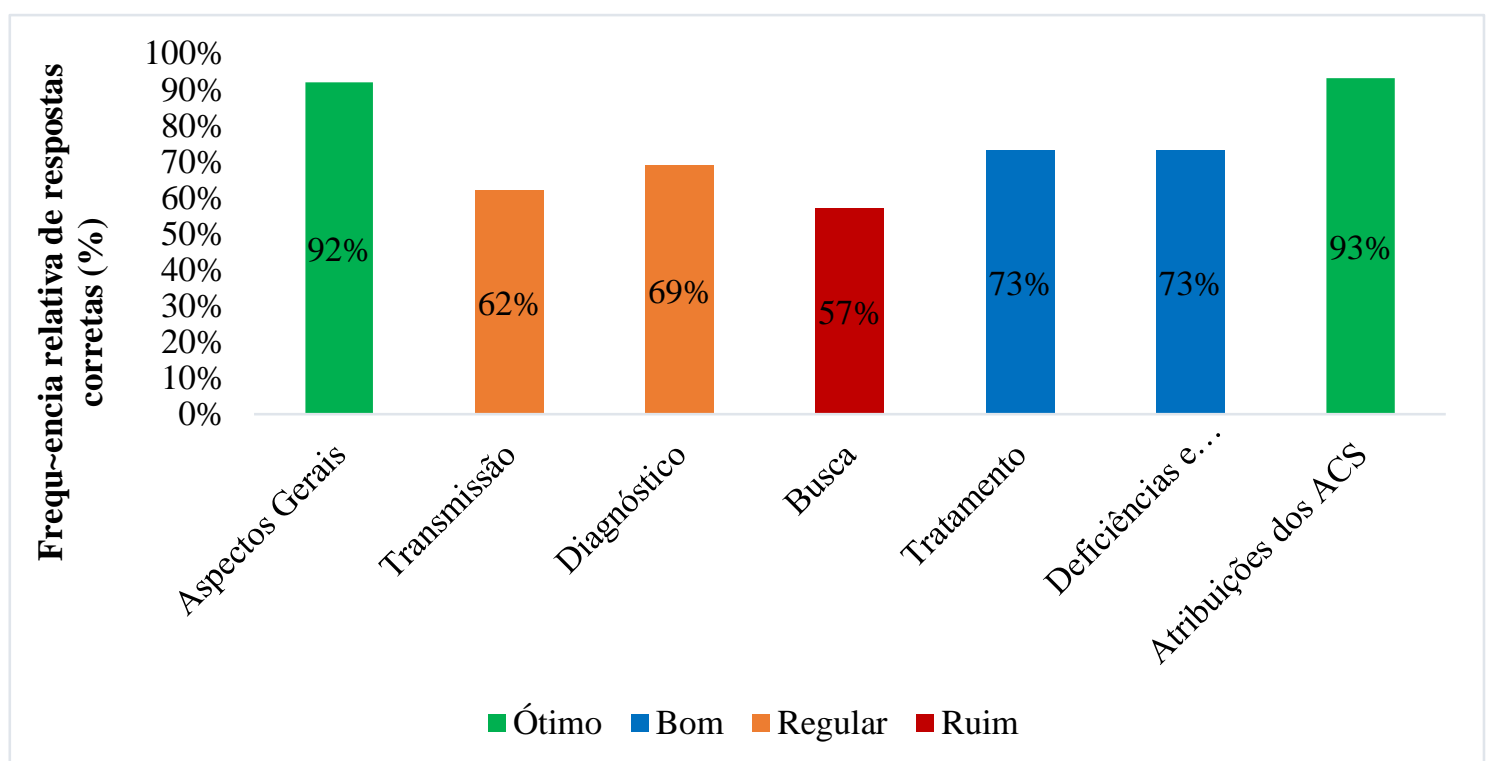

Figura 2 - Frequência relativa de respostas corretas quanto ao conhecimento de agentes comunitários de saúde sobre hanseníase, por blocos. Iguatu, Ceará, 2017.

Quando averiguado o panorama geral da pesquisa, o nível de conhecimento das ACS, no total de acertos do questionário, evidenciou-se bom, pelo percentual de $74 \%$.

Na comparação dos níveis de conhecimento por grupos de escolaridade e estado civil, identificou-se ausência de distribuição normal dos dados aos testes de KolmogorovSmirnov $(0,173 ; p<0,001)$ e Shapiro-Wilk $(0,917 ; p<0,001)$. Teste de Levene reconheceu não existir homogeneidade de variância entre os grupos analisados (Levene escolaridade 
$(4,67)=2,45, p=0,054$; Levene estado civil $(3,68)=2,25, p=0,90)$. Os resultados da ANOVA demonstraram não haver diferenças entre os grupos quanto a escolaridade [Welch's $F(4,9,66)=2,71 ; p<0,09$ ] e quanto a estado civil [Welch's $F(3,9,92)=0,69$; $p=0,57]$.

$\mathrm{O}$ teste $t$ para amostras independentes demonstrou que as ACS que têm ou tiveram pacientes com hanseníase apresentaram nível de conhecimento estatisticamente maior $(M=26,42 ; D P=3,53)$ do que aqueles que não têm ou tiveram $(M=24,41 ; D P=3,85)$ $(t(70)=2,004, p<0,05)$. O tamanho do efeito da diferença entre esses grupos foi médio $(d$ de Cohen=0,56).

Não houve diferença estatisticamente significativa no nível de conhecimento sobre hanseníase entre as ACS que participaram do curso de formação específico da profissão e os que não participaram, bem como entre aqueles que participaram de capacitação sobre o tema e os que não participaram (Tabela 2).

Tabela 2 - Testes de diferenças nos níveis de conhecimento de agente comunitário de saúde que tem/tiveram pacientes com hanseníase, participaram de formação específica para agente comunitário de saúde e participaram de capacitação. Iguatu-Ceará, Brasil, 2017.

\begin{tabular}{|c|c|c|c|c|c|c|c|c|c|}
\hline \multirow{3}{*}{\multicolumn{2}{|c|}{ Grupos analisados }} & \multicolumn{3}{|c|}{ Escores } & \multicolumn{5}{|c|}{ Estatística do teste $t$ (Bootstrapping sample) } \\
\hline & & \multirow{2}{*}{$M^{\dagger}$} & \multirow{2}{*}{$D P^{\ddagger}$} & \multirow{2}{*}{$t^{\S}$} & \multirow{2}{*}{$G l^{\|}$} & \multirow{2}{*}{$p$-valor } & \multirow{2}{*}{$\begin{array}{l}\text { Diferença de } \\
\text { Média }\end{array}$} & \multicolumn{2}{|c|}{$\begin{array}{l}\text { ICII da Diferença de } \\
\text { Média }(95 \%)\end{array}$} \\
\hline & & & & & & & & $\begin{array}{l}\text { Limite } \\
\text { inferior }\end{array}$ & $\begin{array}{l}\text { Limite } \\
\text { superior }\end{array}$ \\
\hline \multirow{2}{*}{$\begin{array}{l}\text { Tem/ } \\
\text { tiveram } \\
\text { pacientes } \\
\text { com } \\
\text { hanseníase }\end{array}$} & Sim & 26,42 & 3,53 & \multirow[b]{2}{*}{2,004} & \multirow[b]{2}{*}{70} & \multirow[b]{2}{*}{$0,04 *$} & \multirow[b]{2}{*}{2,006} & \multirow[b]{2}{*}{0,009} & \multirow[b]{2}{*}{4,004} \\
\hline & Não & 24,41 & 3,85 & & & & & & \\
\hline \multirow{2}{*}{$\begin{array}{l}\text { Capacitação } \\
\text { sobre } \\
\text { hanseníase }\end{array}$} & Sim & 26,44 & 3,72 & \multirow{2}{*}{1,745} & \multirow{2}{*}{70} & \multirow{2}{*}{0,85} & \multirow{2}{*}{1,622} & \multirow{2}{*}{$-0,232$} & \multirow{2}{*}{3,47} \\
\hline & Não & 24,82 & 3,40 & & & & & & \\
\hline \multirow{2}{*}{$\begin{array}{l}\text { Formação } \\
\text { específica }\end{array}$} & Sim & 26,03 & 3,76 & \multirow{2}{*}{0,420} & \multirow{2}{*}{70} & \multirow{2}{*}{0,67} & \multirow{2}{*}{0,463} & \multirow{2}{*}{$-1,738$} & \multirow{2}{*}{2,664} \\
\hline & Não & 25,57 & 3,43 & & & & & & \\
\hline
\end{tabular}

Nota: ${ }^{\dagger} M=$ média; ${ }^{\dagger} D P=$ desvio padrão; ${ }^{\S} t=$ teste $t ;{ }^{\|} G l=$ graus de liberdade; ${ }^{\mathrm{I}} \mathrm{IC}=$ intervalo de confiança; $*=p<0,05$.

\section{Discussão}

Reconheceu-se um nível de conhecimento ótimo das ACS relativo aos aspectos gerais da hanseníase. Entretanto, destacam-se divergências quanto ao agente etiológico causador da doença ser uma bactéria e o fato de alguns participantes afirmarem que se trata de uma doença hereditária.

Em Guarabira-PB, foi verificada a compreensão de 14 ACS em relação à hanseníase, evidenciando que estes reconhecem as manchas como sinais e sintomas clássicos da doença, entretanto, necessitam de um conhecimento mais aprofundado, considerando que 
a doença também acomete o sistema nervoso periférico causando incapacidades permanentes (12).

O trabalho do ACS é constituído por atribuições as quais incluem: cadastro das famílias, mapeamento da comunidade, coleta de informações sobre o diagnóstico demográfico e sociocultural da comunidade, registro dos dados de nascimentos, óbitos, doenças e outros agravos, informação para os usuários sobre datas de consultas e exames e estabelecimento de integração entre equipe de saúde e população adscrita. Entretanto, tem se limitado a produção e preenchimento de dados e informações, podendo desencadear uma descaracterização do trabalho do ACS ao limitar seu campo de trabalho a ações mais pontuais (13).

O processo formativo junto a esta categoria de trabalho é inacabado e progressivo, pois o profissional precisa estar apto a atender às especificidades e complexidades exigidas pela comunidade. Dentre as atribuições a serem exigidas pelo ACS, encontra-se a articulação dos conhecimentos científicos e populares com vistas a construção de um projeto terapêutico compartilhado (14).

O homem é considerado a única fonte de infecção, não ocorrendo transmissão hereditária ou através de relações sexuais (15). Um estudo realizado com 179 ACS dos municípios de Pouso Alegre e Poços de Caldas em Minas Gerais, trouxe que 90,2\% dos entrevistados consideraram a hanseníase uma doença contagiosa (15), o que corrobora com os achados desta pesquisa.

Os participantes apresentaram um conhecimento deficiente em relação à classificação operacional da doença: paucibacilar e multibacilar. Depreende-se uma deficiência dos saberes empíricos que pode estar associada ao estigma e preconceito sobre a forma de transmissão (16).

Destarte a estes dados, é válido que ações em educação permanente sejam realizadas com vistas a não só disseminar o conhecimento adequado como também desmistificar tabus e informações incoerentes que possam levar a segregação e preconceito aos pacientes (2). A doença possui uma carga segregacionista milenar, pois desde o aparecimento dos primeiros casos os doentes eram caracterizados como impuros, desfigurados e portadores de uma doença incurável. Tal caracterização desencadeou ações sanitárias errôneas que culminaram no aumento do preconceito e exclusão, como o caso do isolamento compulsório de pessoas infectadas e suas famílias (17).

Sabe-se que a transmissão da hanseníase ocorre pelo contato íntimo e prolongado de um indivíduo susceptível com paciente bacilífero sem tratamento. Após a confirmação do caso, a classificação operacional do tratamento é baseada no número de lesões, sendo: Paucibacilar (PB) - casos com até cinco lesões de pele, e Multibacilar (MB) - casos com seis ou mais lesões de pele (15).

Essa classificação visa definir o esquema terapêutico da doença. Quando possível, fazse necessário a realização da baciloscopia de pele no auxílio do diagnóstico, sendo que seu resultado positivo classifica o paciente como multibacilar, porém o resultado negativo não exclui o diagnóstico da hanseníase (2).

A classificação operacional da doença se relaciona ao diagnóstico precoce e tratamento eficaz, que são medidas necessárias para a redução de danos e prevenção de incapacidades físicas. O diagnóstico da hanseníase é essencialmente realizado pela história clínica e epidemiológica com auxílio do exame dermatoneurológico (2). Um estudo avaliou o conhecimento de 43 ACS sobre a hanseníase e detectou pior desempenho sobre o diagnóstico, obtendo 54,65\% de acertos. Ressaltaram ainda, falta de informação acerca dos métodos de identificação e confirmação diagnóstica, classificação operacional baseada no diagnóstico e diagnóstico diferencial (8).

Em relação a busca, foram evidenciados conhecimentos insatisfatórios dos 
participantes, principalmente em relação as medidas de controle dos contatos e busca ativa. Pesquisa em Teresina-PI para verificar a atuação dos ACS na prevenção e controle da hanseníase identificou que as medidas de prevenção mais citadas pelos participantes foram o encaminhamento dos suspeitos para as unidades básicas de saúde e avaliação da necessidade da vacina Bacilo de Calmette e Guérin (BCG) (18). Destaca-se, portanto, que estas atividades fazem parte da busca ativa, atividade indispensável para a vigilância em saúde.

Acerca das medidas de controle de contatos de hanseníase, os dados do presente estudo se opõem a resultados que discorrem que os ACS devem realizar busca ativa de faltosos e de contatos intradomiciliares e fazer supervisão da dose medicamentosa no domicílio, quando solicitado (13).

No que tange ao conhecimento sobre o tratamento, um perfil semelhante foi encontrado no estudo realizado em Cocal-PI, onde quase a totalidade dos ACS reconheceram que o tratamento deve ser realizado por completo, mesmo diante da ausência de sintomas e de modo supervisionado. Afirma-se ainda, que os ACS conhecem o esquema terapêutico utilizado, principalmente para a forma multibacilar, se opondo aos dados desta pesquisa onde as ACS demonstraram um percentual maior de erros/dúvidas (19).

Dessa forma, verifica-se uma deficiência quanto à classificação operacional da doença, uma possível incompreensão acerca das formas clínicas e fragilidade quanto a orientações dos fármacos. No entanto, nas demais respostas sobre tratamento, as ACS identificaram que devem atuar em conjunto com os demais membros da equipe de saúde da família e reconhecem a cura da enfermidade através de um tratamento eficaz e completo.

É de suma importância que o ACS conheça a duração do tratamento, visto que deverá acompanhar os pacientes durante toda a terapêutica, incentivando-os a não desistir, prevenindo recidivas, estigmas, incapacidades, bem como investigando possíveis novos casos (8). Acrescenta-se que o profissional deve conhecer, também, os fármacos e as possíveis reações, uma vez que as cartelas são diferentes quanto aos fármacos, quantidade e dosagem, e o surgimento de reação adversa não é incomum.

A hanseníase tem cura, todavia, se não diagnosticada e tratada precocemente pode trazer graves impactos para os pacientes e seus familiares, pelas sequelas que, na maioria das vezes, evoluem para incapacidades físicas. A incapacidade não se resume apenas a disfunção física, engloba limitações de atividades, preconceitos, discriminação, e restrição à participação social dos indivíduos $(18,20)$.

Além disso, é fundamental que haja o acompanhamento dos casos de alta por cura do tratamento da hanseníase mesmo após os cinco anos de avaliações nos serviços de saúde, com agendamento de acordo com cada caso, diante do surgimento de nova lesão de pele e/ou dores nos nervos periféricos ou se apresentarem piora na função motora e/ou sensitiva, sendo indispensável uma avaliação neurológica simplificada no momento dessa consulta (2).

Sobre o conhecimento dos ACS quanto suas atribuições no desenvolvimento destas ações, reconheceu-se um nível ótimo fortalecendo a compreensão de que nas ações de combate e controle da hanseníase, cada membro da equipe tem sua função, sendo uma responsabilidade comum a todos os profissionais o desenvolvimento de estratégias de educação em saúde. Dessa forma, todos devem estar qualificados para conduzir informações para a família e comunidade (21).

O profissional ACS atua como mediador entre o serviço de saúde e a população, dessa forma possui como função conhecer as especificidades do seu território e os casos que implicam um maior cuidado, como é o caso da hanseníase. Assim, as capacitações pautadas na política da educação permanente sobre enfermidades que acometem a comunidade, são primordiais para o bom desempenho destes profissionais (21). 
A educação permanente em saúde apresenta-se como um instrumento operacionalizador de mudança na concepção e prática dos atores que operam e conduzem os serviços de atenção primária. Sob a ótica da incorporação do modelo integral em saúde e de políticas que promovem saúde, a educação permanente estabelece uma interseção do trabalho com o aprendizado em ato, permeando o cotidiano dos ACS e estimulando a autonomia dos sujeitos através de uma avaliação crítica e reflexiva da realidade (22).

Apontam-se como limitações deste estudo: a utilização de um instrumento não validado e autopreenchido e o fato de a coleta de dados ter sido realizada somente na zona urbana, devido a dificuldades logísticas e operacionais encontradas pelos pesquisadores.

\section{Considerações finais}

Identificou-se um nível de conhecimento satisfatório sobre a hanseníase, nos quesitos: aspectos gerais, atribuições dos agentes comunitários de saúde, tratamento, incapacidades físicas. Entretanto, os itens transmissão, diagnóstico e busca tiveram avaliações negativas, demonstrando a necessidade de articulações futuras acerca do preparo e instrução do ACS na abordagem ao usuário da comunidade afetado pela hanseníase.

Ressalta-se a preocupação com as necessidades de aprendizagem evidenciadas por algumas respostas incorretas obtidas. Com isso, sugere-se o fomento de ações em educação permanente junto a estas profissionais. Os ACS integram o serviço, devendo os demais atores envolvidos nessa rede de cuidados no âmbito da Estratégia Saúde da Família também incorporarem suas atribuições para que a prática profissional seja cada vez mais exitosa e respaldada em evidências atuais e vigentes.

\section{Referências}

1. Brasil. Ministério da Saúde. Secretaria de Vigilância em Saúde. Departamento de Vigilância das Doenças Transmissíveis. Guia prático sobre a hanseníase [recurso eletrônico]. Brasília: Ministério da Saúde, $2017 . \quad$ Disponível em: https://portalarquivos2.saude.gov.br/images/pdf/2017/novembro/22/Guia-Pratico-de-HanseniaseWEB.pdf

2. Brasil. Ministério da Saúde. Diretrizes para vigilância, atenção e eliminação da Hanseníase como problema de saúde pública: manual técnico-operacional. Brasília: Ministério da Saúde, 2016. Disponível em: http://portalarquivos2.saude.gov.br/images/pdf/2016/fevereiro/04/diretrizeseliminacao-hanseniase-4fev16-web.pdf

3. World Health Organization. Global Leprosy Strategy: Accelerating towards a leprosy-free world. (Period 2016-2020). Nova Délhi: OMS-SEARO, 2016. Disponível em: https://apps.who.int/iris/handle/10665/254907

4. Brito KKG, Araújo DAL, Uchôa REMN, Ferreira JDL, Soares MJGO, Lima JO. Epidemiologia da hanseníase em um estado do nordeste brasileiro. Rev enferm UFPE on line. 2014; 8(8):2686-93.

5. World Health Organization. Global leprosy (Hansen disease) update, 2019: time to step-up prevention initiatives. Weekly epidemiological Record. 2020 [citado em 2021 jun 09]; 95(36): 417-440. Disponível em: https://reliefweb.int/sites/reliefweb.int/files/resources/WER9536-eng-fre.pdf

6. Brasil. Ministério da Saúde. Sala de Apoio à Gestão Estratégica. Situação de Saúde: Hanseníase. Brasília, 2017. Disponível em: http://sage.saude.gov.br/\#.

7. Alonso CMC, Béguin PD, Duarte FJCM. Atuação do agente comunitário de saúde na Estratégia Saúde da Família: metassíntese. Rev saúde pública 2018; 52(14).

8. Silva JCA, Ribeiro MDA, Oliveira SB. Avaliação do nível de informação sobre Hanseníase dos Agentes Comunitários de Saúde. Rev Bras Promoç Saúde 2016; 29(3): 364-70.

9. IBGE - Instituto Brasileiro de Geografia e Estatística. Censo Demográfico 2010 - Características Gerais da População. Resultados da Amostra. Rio de Janeiro, 2016. Disponível em: https://cidades.ibge.gov.br/v4/brasil/ce/iguatu/panorama.

10. Brasil. Ministério da Saúde. Sala de Apoio à Gestão Estratégica. Situação de Saúde: Hanseníase. Brasília, 2017. Disponível em:http://sage.saude.gov.br/\#.

11. Brasil. Departamento da Atenção Básica. Portal do Departamento de Atenção Básica. Histórico de 
Vittalle - Revista de Ciências da Saúde v. 33, n. 2 (2021) 48-58

Cobertura da Saúde da Família. Brasília, 2017. Disponível em: http://dab.saude.gov.br/dab/historico_cobertura_sf/historico_cobertura_sf_relatorio.php.

12. Andrade CG, Costa ICP, Freire MEM, Santos KFO, Gouveia EML, Claudin HG. Hanseníase: Compreensão de Agentes Comunitários de Saúde. Rev bras ci Saúde. 2011; 15(1): 17-24.

13. Morosini MVG, Fonseca AF, Lima LD. Política Nacional de Atenção Básica 2017: retrocessos e riscos para o Sistema Único de Saúde. Saúde debate. 2018; 42(116):11-24.

14. Secco, AC, Rodrigues PM, Ledur CS, Zanatta E, Mozzaquatro CO, Arpini DO. Educação Permanente em Saúde para Agentes Comunitários: um Projeto de Promoção de Saúde. Gerais, Rev Interinst Psicol. 2020; 13(1): 1-17.

15. Mesquita MF, Gomes CFL. Preconceito e conhecimento sobre hanseníase: a situação do agente comunitário de saúde. Revista Bioethikos. 2014; 8(2): 153-60.

16. Oliveira CM, Linhares MSC, Ximenes Neto FRG, Mendes IMVP, Kerr LRFS. Conhecimento e práticas dos agentes comunitários de saúde sobre hanseníase em um município hiperendêmico. Saúde Ver. 2018; 18(48): 39-50.

17. Pinheiro, MGC, Simpson CA. Preconceito, estigma e exclusão social: trajetória de familiares influenciada pelo tratamento asilar da hanseníase. Rev enferm UERJ. 2017; 25: e13332.

18. Santos AR, Ignotti E. Prevenção de incapacidade física por hanseníase no Brasil: análise histórica. Ciência \& Saúde Coletiva. 2020; 25(10): 3731-744.

19. Araújo DYML, Andrade JS, Madeira MZA. A atuação dos agentes comunitários de saúde do município de Teresina/Piauí sobre hanseníase. Rev Rene 2011; 12(n.esp.): 995-1002.

20. Souza AE, Martins MGT. Aspectos afetivos e comportamentais do portador de hanseníase frente ao estigma e preconceito. Revista de Iniciação Científica da Universidade Vale do Rio Verde, Três Corações. 2018; 8(1): 104-113.

21. Sales JCS, Luz VLES, Branco FMFC, Araújo MFF, Castro SHTS, Silva TCA. O significado da hanseníase para o agente comunitário de saúde. Revista Interdisciplinar. 2013; 6(1): 17-24.

22. Siega CK, Vendruscolo C, Zanatta EA. Educação permanente com agentes comunitários de saúde para instrumentalização da visita domiciliar: relato de experiência. Rev Enferm Contemp. 2020; 9(1): 94-100. 